University of Nebraska - Lincoln

DigitalCommons@University of Nebraska - Lincoln

\title{
Isolation and Enrichment of Abundant Microsatellites from a Channel Catfish (Ictalurus punctatus) Brain cDNA Library
}

\author{
Dan Nonneman \\ USDA, ARS, U.S.Meat Animal Research Center, Clay Center, Nebraska \\ Geoffrey C. Waldbieser \\ USDA, ARS, Catfish Genetics Research Unit, Thad Cochran National Warm Water Aquaculture Center
}

Follow this and additional works at: https://digitalcommons.unl.edu/usdaarsfacpub

Part of the Agricultural Science Commons

Nonneman, Dan and Waldbieser, Geoffrey C., "Isolation and Enrichment of Abundant Microsatellites from a Channel Catfish (Ictalurus punctatus) Brain cDNA Library" (2005). Publications from USDA-ARS / UNL Faculty. 284.

https://digitalcommons.unl.edu/usdaarsfacpub/284

This Article is brought to you for free and open access by the U.S. Department of Agriculture: Agricultural Research Service, Lincoln, Nebraska at DigitalCommons@University of Nebraska - Lincoln. It has been accepted for inclusion in Publications from USDA-ARS / UNL Faculty by an authorized administrator of DigitalCommons@University of Nebraska - Lincoln. 


\section{ISOLATION AND ENRICHMENT OF ABUNDANT MICROSATELLITES FROM A CHANNEL CATFISH (ICTALURUS PUNCTATUS) BRAIN cDNA LIBRARY}

\section{Dan Nonneman}

USDA, ARS, U.S. Meat Animal Research Center, Clay Center, Nebraska, USA

\section{Geoffrey C. Waldbieser}

USDA, ARS, Catfish Genetics Research Unit, Thad Cochran National Warm Water Aquaculture Center, Stoneville, Mississippi, USA

Efforts to construct a genetic linkage map of channel catfish have involved identification of random genomic microsatellite markers, as well as anchored Type I loci (expressed genes) from channel catfish. To identify Type I markers we constructed a directional cDNA library from brain tissue to obtain expressed catfish sequences that could be used for single nucleotide polymorphism (SNP) marker development. These cDNA sequences surprisingly contained a high proportion of microsatellites (about 14\%) in noncoding regions of expressed sequence tags (ESTs), many of which were not associated with known sequences. To further identify cDNAs with microsatellites and reduce the number of sequencing reactions needed for marker development, we enriched this library for repeat sequences and sequenced clones from both directions. A total of 1644 clones from seven repeat-enriched captures (CA, GT, CT, GA, MTT, TAG, and TAC) were sequenced from both ends, and 795 nonredundant clones were assembled. Thirty-seven percent of the clones contained microsatellites in the trimmed sequence. After assembly in the TIGR Catfish Gene Index (CfGI), 154 contigs matched known vertebrate genes and 92 contigs contained microsatellites. When BLAST-matched orthologues were available for similarity alignments, $28 \%$ of these contigs contained repeats in the $5^{\prime}$-UTR, $72 \%$ contained repeats in the $3^{\prime}$-UTR, and $8 \%$ contained repeats at both ends. Using biotinylated repeat oligonucleotides coupled with streptavidin-coated magnetic beads, and rapid, single-pass hybridization, we were able to enrich our plasmid library greater than two-fold for repeat sequences and increase the ability to link these ESTs with known sequences greater than six-fold.

Keywords: Channel catfish; Microsatellites; Expressed sequences; Brain

\section{INTRODUCTION}

Genetic improvement of livestock requires a dedicated breeding program that relies on the determination of traits that are desirable for future generations. Identification and incorporation of these traits into subsequent generations can be difficult

Mention of trade names or commercial products is solely for the purpose of providing information and does not imply recommendation, endorsement, or exclusion of other suitable products by the U.S. Department of Agriculture.

Address correspondence to Dan Nonneman, USDA, ARS, U.S. Meat Animal Research Center, Spur 18D, P.O. Box 166, Clay Center, NE 68933-0166, USA. E-mail: nonneman@email.marc.usda.gov 
and time-consuming based upon phenotype alone. Therefore, marker assisted selection (MAS) of a trait or group of traits is highly desirable and can be achieved through the development of an informative genetic linkage map $(1,2)$. Historically, linkage maps have been developed from random microsatellite markers (Type II markers) that are highly polymorphic and rapidly obtained (3-5), but have little or no comparative value to unrelated species. Anchored loci (Type I markers) are usually then assigned to the map to identify homologous regions of chromosomes between species and further the information obtained with Type II loci (6-9). Ideally, a genetic linkage map would consist of a great number of highly polymorphic, known (Type I) anchored loci (10). However, microsatellite markers in cDNAs of most species are estimated to range from 0.1 to $1 \%$ and are therefore not very plentiful for genetic linkage maps (11-13). The degree of polymorphism for Type I and Type II markers varies between species and the reference population from which it is obtained. We constructed a cDNA library from channel catfish brain mRNA and sequenced these clones to identify known genes and find markers that would allow us to place these anchored loci on the catfish genetic linkage map (14).

\section{MATERIALS AND METHODS}

\section{Channel Catfish Brain Primary cDNA Library}

Brain tissue was obtained from five juvenile USDA103-strain channel catfish at Stoneville, MS, during the month of November, and RNA was extracted using Trizol reagent (Life Technologies, Carlsbad, CA, USA). A channel catfish brain cDNA library was directionally cloned into NotI-SalI cloning sites of pSport1 following the protocol for the SuperScript Plasmid cDNA Library kit (Life Technologies, Carlsbad, CA, USA). After transformation into DH5 $\alpha$ cells and growth on LB-agar, colonies were grown in $0.1 \mathrm{ml} \mathrm{LB}$ media and stored as glycerol stocks at $-80^{\circ} \mathrm{C}$.

\section{DNA Sequencing and Analysis}

Frozen colonies were replicated into $0.35 \mathrm{ml}$ 2XYT media in $0.6 \mathrm{ml}$ deep well plates and cultured overnight in a HiGro shaking incubator (GeneMachines, San Carlos, CA). Bacteria were pelleted by centrifugation in an Eppendorf 5810R centrifuge equipped with an A-2-DWP rotor (Brinkmann Instruments, Inc., Westbury, $\mathrm{NY}$ ) for $30 \mathrm{~min}$ at $1900 \times \mathrm{g}$. Bacterial pellets were resuspended in $75 \mu \mathrm{l}$ of solution P1 containing $100 \mu \mathrm{g} / \mathrm{ml} \mathrm{RNase} \mathrm{A} \mathrm{(Qiagen,} \mathrm{Chatsworth,} \mathrm{CA)} \mathrm{for} 5 \mathrm{~min}$, lysed with $75 \mu \mathrm{l}$ solution of $\mathrm{P} 2$ for $5 \mathrm{~min}$, and neutralized with $105 \mu 1$ solution of P3. The plate was centrifuged at $1900 \times \mathrm{g}$ for $10 \mathrm{~min}$. Two hundred microliters of supernatant were transferred to a $0.45 \mu \mathrm{m}$ hydrophobic PVDF filter plate (Whatman Inc., Clifton, NJ) with wide bore tips and mixed with $30 \mu \mathrm{l}$ Procipitate (Ligochem, Fairfield, NJ), which was preloaded onto the filter plate. After a $5 \mathrm{~min}$ room temperature incubation, the supernatant was filter-centrifuged into a $0.6 \mathrm{ml}$ deep well receiver plate $20 \mathrm{~min}$ at $1900 \times \mathrm{g}$. Two hundred microliters of isopropanol was added to the filtered supernatant, mixed vigorously, and centrifuged $30 \mathrm{~min}$ at $1900 \times \mathrm{g}$. Precipitated DNA was washed with $100 \mu 170 \%$ ethanol, centrifuged $5 \mathrm{~min}$ at $1900 \times \mathrm{g}$, inverted to remove the ethanol, and air-dried. The plasmid DNA was resuspended in $40 \mu 1$ 
water, and $400 \mathrm{ng}$ was sequenced using $1 / 16$ dilution $(0.5 \mu \mathrm{l})$ of BigDye Terminator Cycle Sequencing Ready Reaction Kit or dGTP BigDye Terminator Cycle Sequencing Ready Reaction Kit (Applied Biosystems, Foster City, CA, USA), halfBD Dye Terminator Sequencing Reagent (Sigma, St. Louis, MO, USA), 5\% dimethysulfoxide, and 4pmol of T7 or SP6 primer in a total reaction volume of $5 \mu 1$. Reactions were precipitated with $20 \mu 170 \%$ isopropanol, washed with $75 \%$ ethanol, resuspended in $10 \mu \mathrm{l}$ water, and sequences were determined on an ABI PRISM 3700 DNA Analyzer (Applied Biosystems, Foster City, CA).

Base calling was performed using Phred v0.000925.c $(15,16)$. Sequences were processed with Lucy (17) to remove vector and delete sequences with less than 100 Phred20 bases. Before submission to GenBank, mitochondrial and ribosomal RNA transcripts were removed by BlastN (18) screening of the high-quality sequences against a database containing the channel catfish mitochondrial genome and ribosomal sequences. Catfish sequences were downloaded from the TIGR catfish gene index, and microsatellites were identified using Sputnik (www.abajian.net/ sputnik) using six repeats as a cutoff score. The TCs were normalized by grouping of clones with identical gene match and/or grouping of clones with separate $5^{\prime}$ and $3^{\prime}$ reads that had different $\mathrm{TC}$ designations. The number of microsatellites in expressed sequences of other species was determined by BlastN of the TIGR gene indices (www.tigr.org/tdb/tgi) without a filter, expected threshold of 100, and a word length of 11 .

\section{Microsatellite-Enriched Libraries}

Microsatellite capture was performed using the GeneTrapper kit (Life Technologies, Carlsbad, CA, USA) and a biotinylated oligonucleotide for hybridization of the total amplified cDNA library. Seven separate enrichments were performed using $\mathrm{dCA}_{9}, \mathrm{dGT}_{9}, \mathrm{dCT}_{9}, \mathrm{dGA}_{9}, \mathrm{dMTT}_{6}, \mathrm{dTAG}_{6}$, and $\mathrm{dTAC}_{6}$ oligonucleotides. After capture, repair with $\mathrm{T} 7$ primer, and transformation, the clones were sequenced as above using T7 and SP6 primers. Individual colonies of each enriched library were evaluated for presence of the microsatellite repeat by PCR amplification. The reactions used $0.5 \mu \mathrm{l}$ of a $2 \mathrm{~h}$ bacterial culture in $2 \mathrm{XYT}$ media, with $5 \mathrm{pmol}$ each of the nonbiotinylated repeat oligonucleotide and the downstream SP6 primer in a total reaction volume of $10 \mu \mathrm{l}$. Reaction conditions were 35 cycles of $94^{\circ} \mathrm{C}$ for $30 \mathrm{sec}$, $57^{\circ} \mathrm{C}$ for $45 \mathrm{sec}$, and $72^{\circ} \mathrm{C}$ for $1.5 \mathrm{~min}$ in a PTC-200 thermalcycler (M.J. Research, Inc., Waltham, MA). Reactions were analyzed by agarose gel electrophoresis and ethidium staining.

\section{Genotype Analysis}

Genotyping primers were constructed using GeneRunner (Hastings Software, Hastings-on-Hudson, NY) or Primer3 v.0.9 $(19,20)$ and purchased from Resgen (now Invitrogen, Carlsbad, CA). A 19-bp 5' extension was added to one primer of the set to facilitate fluorescent labeling of the amplification product (5'-CACGACGTTGTAAAACGAC-3'), and a fluorescent primer with this sequence was synthesized containing 5' 6-FAM or NED labels (Applied Biosystems). The $15 \mu \mathrm{l}$ genotyping reactions contained $10 \mathrm{mM}$ Tris- $\mathrm{HCl}[\mathrm{pH} 9.0], 50 \mathrm{mM} \mathrm{KCl}, 0.1 \%$ Triton 
$\mathrm{X}-100,1 \mathrm{mM} \mathrm{MgCl}_{2}, 67 \mu \mathrm{M}$ dNTPs, $0.5 \mathrm{U}$ Taq polymerase (Promega Corporation, Madison, WI) 3 pmoles of the extended primer, 3 pmoles of the fluorescent 19-mer, 6 pmoles of the nonextended primer, and $40 \mathrm{ng}$ genomic DNA. The reaction profile for all loci was $95^{\circ}$ for $3 \mathrm{~min}, 2$ cycles of $95^{\circ}$ for $1 \mathrm{~min}$ and $50^{\circ}$ for $30 \mathrm{sec}$, then 23 cycles of $95^{\circ}$ for $30 \mathrm{sec}$ and $50^{\circ}$ for $30 \mathrm{sec}$, then incubation at $72^{\circ}$ for $4 \mathrm{~min}$ in a PTC-200 thermalcycler (MJ Research, Watertown, MA). One microliter of reaction product was mixed with $9 \mu \mathrm{l} \mathrm{Hi-Dye} \mathrm{formamide} \mathrm{and} 0.2 \mu \mathrm{l}$ GeneScan-400HD [ROX] size standard (Applied Biosystems). Alleles from two linkage map reference families were determined on the ABI PRISM 3700 DNA Analyzer with GeneScan software (Applied Biosystems).

\section{RESULTS}

\section{Channel Catfish Brain Primary cDNA Library}

A cDNA library from channel catfish adult brain was constructed in DH5 cells that contained approximately 500,000 individual clones with an average insert size of $1.8 \mathrm{~kb}$ (ranging from 0.2 to $4.7 \mathrm{~kb}$ ). Direct sequencing of 1632 primary clones resulted in 1219 high-quality trimmed sequences of which $16 \%$ of these clones were redundant and $6 \%$ contained mitochondrial sequences, resulting in 882 unique clones. More than $80 \%$ of the clones sequenced had quality sequence of greater than $500 \mathrm{bp}$ and some contained quality sequence to $1 \mathrm{~kb}$, with an overall average read length of $427 \mathrm{bp}$ (Table 1). Sequencing failures were caused by more than one clone per well, lack of growth, or a sequence compression due to a poly (dG-dC) or microsatellite immediately following the cloning/primer site.

In the primary library, $13 \%$ of the $5^{\prime}$-end sequences matched to a known sequence in GenBank (Table 1). Fourteen percent of the catfish sequences contained microsatellites with repeat motifs greater than six dinucleotides, trinucleotides, or tetranucleotides. The most common repeat motif in the microsatellite-containing clones was CA/GT $(29 \%)$ followed by AAT/ATT $(25 \%)$. Other repeat motifs identified in the catfish cDNAs were AG/CT, AT/TA, AAC/GTT, ACC/GGT, ACG/CGT, AGG/CCT, ATG/CAT, CAC/GTG, AAAC/GTTT, AAAG/CTTT,

Table 1 Comparison of unique clones from primary and microsatellite-enriched brain cDNA libraries

\begin{tabular}{lccr}
\hline & Match & No match & Total \\
\hline Primary library & & & \\
$\quad$ Microsatellite & $14^{a}$ & 112 & 126 \\
No microsatellite & $103^{b}$ & 653 & 756 \\
Total & 117 & 765 & 882 \\
Enriched library & & & \\
Microsatellite & 92 & 202 & 294 \\
No microsatellite & 62 & 439 & 501 \\
Total & 154 & 641 & 795 \\
\hline
\end{tabular}

${ }^{a} 9$ clones common to both libraries.

${ }^{b} 43$ clones common to both libraries. 
Table 2 and Table 3 Frequency of dinucleotide repeats in animal ESTs

\begin{tabular}{lccccc}
\hline Species & Sequences $^{a}$ & $\mathrm{CA}_{20}(\%)$ & $\mathrm{AT}_{20}(\%)$ & $\mathrm{CT}_{20}(\%)$ & Total (\%) \\
\hline Catfish & 7,764 & 8.15 & 2.24 & 3.56 & 14.02 \\
Salmon & 24,783 & 2.94 & 1.36 & 1.34 & 5.63 \\
Zebrafish & 74,236 & 3.83 & 0.89 & 0.89 & 5.61 \\
Trout & 40,628 & 2.73 & 0.97 & 1.02 & 4.72 \\
Mouse & 669,402 & 1.85 & 0.32 & 0.69 & 2.86 \\
Cattle & 89,722 & 0.98 & 0.43 & 0.36 & 1.77 \\
Pig & 58,623 & 0.85 & 0.47 & 0.26 & 1.58 \\
Chicken & 11,742 & 0.48 & 0.72 & 0.30 & 1.50 \\
Human & 843,769 & 0.74 & 0.19 & 0.27 & 1.20 \\
\hline
\end{tabular}

${ }^{a}$ Totally unique sequences assembled by TIGR (http://www.tigr.org).

CTTT, AAAT/ATTT, and AATC/GATT (Table 2). Therefore, an enrichment of CA, GT, CT, GA, MTT, TAG, and TAC repeats was chosen to select for cDNAs that contained microsatellites, since 400-600 CA/GT repeat microsatellite sequences were estimated to be present in the library.

\section{Microsatellite-Enriched cDNA Library}

After enrichment of the brain library for CA/GT repeats using a biotinylated $(\mathrm{CA})_{9}$ or $(\mathrm{GT})_{9}$ primer and streptavidin-coated magnetic beads, PCR amplification of transformed clones using the nonbiotinylated $(\mathrm{CA})_{9}$ primer and SP6 vector primer resulted in $88 \%$ positive amplification products of clones, and amplification products ranged from 0.3 to $3.0 \mathrm{~kb}$. The size of these products supported our original observation that the microsatellite sequences resided in both the $5^{\prime}$ and $3^{\prime}$ regions of these cDNAs. A total of 1644 clones from 7 repeat-enriched captures (CA, GT, CT, GA, MTT, TAG, and TAC) were sequenced from both ends using T7 and SP6 primers, and 795 nonredundant clones were assembled with an average read length of $442 \mathrm{bp}$. The redundancy of the enriched library was $51 \%$. Thirty-seven percent of the unique clones (294) contained microsatellites in the vector trimmed sequence (Table 1). The number of unique clones with microsatellites increased 2.2-fold (126 vs. 294), but the number of unique clones containing microsatellites with a GenBank match increased 6.6-fold (14 vs. 92) with enrichment. The enriched library contained 102 new sequences not found in the primary library; 83 of these contained a microsatellite in the sequenced DNA. After assembly in the TIGR Catfish Gene Index (CfGI), 154 (19\%) clones were contained in contigs matching known vertebrate genes or ESTs (Table 3), of which 92 contained microsatellites. Twentyeight percent of the repeats were identified in the $5^{\prime}$-UTR, $72 \%$ in the $3^{\prime}$-UTR, and $8.8 \%$ contained repeats at both ends of the contigs (Table 3 ). This library is listed in the TIGR Catfish Gene Index (CfGI) (http://www.tigr.org/tdb/tgi/cfgi/) as library \#9VO. Sixty-four clones contained more than one repeat motif, typically two different dinucleotide repeats, although tri- and tetranucleotide repeats were also associated with dinucleotide repeats in the same clone and could be captured using different probes. For example, one clone selected with a CT probe 
Table 3 Channel catfish unique EST TIGR contigs (TCs) with sequence identity to known genes

\begin{tabular}{|c|c|c|c|}
\hline Contig $^{1}$ & Gene match & Repeat type ${ }^{2}$ & Location $^{3}$ \\
\hline TC3038 & 14-3-3 protein & AAAT & $3^{\prime}$ \\
\hline TC3802 & $28 \mathrm{kDa}-2$ apolipoprotein & & \\
\hline TC3786 & $42 \mathrm{Sp} 48$ & & \\
\hline TC3091 & Actin-related protein $2 / 3$ complex subunit 4 & & \\
\hline TC4193 & Adenylosuccinate synthetase 2 & $\mathrm{CA}$ & $3^{\prime}$ \\
\hline TC3878 & Aldolase C & GT & $3^{\prime}$ \\
\hline TC3545 & Alpha-2-macroglobulin-3 & & \\
\hline TC4492 & Aminopeptidase & $\mathrm{CA}$ & $3^{\prime}$ \\
\hline TC3400 & Aminopeptidase $\mathrm{N}$ & $\mathrm{CA}$ & $3^{\prime}$ \\
\hline TC3144 & Amisyn & CCA, GT & $3^{\prime}$ \\
\hline TC4400 & Amylase & & \\
\hline TC3220 & AP-3 complex sigma3B subunit & $\mathrm{CT} ; \mathrm{GT}$ & $5^{\prime} ; 3^{\prime}$ \\
\hline TC3303 & ATP citrate lyase & $\mathrm{CA}$ & $3^{\prime}$ \\
\hline TC4014 & Autophagy protein 5-like & CT & $3^{\prime}$ \\
\hline TC4422 & BAB15607, unnamed protein product & & \\
\hline TC3268 & Beta tubulin & $\mathrm{CA}$ & $5^{\prime}$ \\
\hline TC2953 & Beta-actin & & \\
\hline TC3693 & CAC22531. unnamed protein product & $\mathrm{CA}$ & $3^{\prime}$ \\
\hline TC4175 & Calmodulin & GT & $3^{\prime}$ \\
\hline ТC4660 & Casein kinase II, alpha chain & $\mathrm{CA}$ & $3^{\prime}$ \\
\hline TC3570 & Catalase & & \\
\hline TC3951 & $\mathrm{Cd} 81$ & $\mathrm{CA}$ & $3^{\prime}$ \\
\hline TC4317 & $\begin{array}{l}\text { Chaperonin-containing T-complex } \\
\text { protein } 1 \text { eta subunit }\end{array}$ & CA & $3^{\prime}$ \\
\hline TC3245 & chS-Rex-s & $\mathrm{CA}$ & $3^{\prime}$ \\
\hline TC4474 & Chymotrypsinogen 2 & & \\
\hline TC4376 & Clusterin precursor & & \\
\hline TC3869 & Cofilin, muscle isoform & & \\
\hline TC3935 & Cold-inducible RNA binding protein 2 & & \\
\hline TC4413 & Complement C4B & & \\
\hline TC4405 & Cullin homolog 1 & $\mathrm{GT}, \mathrm{CT}$ & $3^{\prime}$ \\
\hline TC4030 & $\begin{array}{l}\text { Cytosolic malate dehydrogenase } \\
\text { thermostable form }\end{array}$ & CT & $3^{\prime}$ \\
\hline TC4084 & Diazepam binding inhibitor & & \\
\hline TC4498 & dJ347H13.4 (novel protein) & $\mathrm{CA}$ & $3^{\prime}$ \\
\hline TC4563 & DMalpha2b & CA; GT & $5^{\prime} ; 3^{\prime}$ \\
\hline TC4002 & DMgamma1 & & \\
\hline TC4458 & Dok4 & GT & $5^{\prime}$ \\
\hline TC4639 & $\begin{array}{l}\text { Downstream Regulatory Element-Antagonist } \\
\text { Modulator }\end{array}$ & $\mathrm{GT}, \mathrm{CT}$ & $3^{\prime}$ \\
\hline TC3102 & Dynein light chain 2 & $\mathrm{CA} ; \mathrm{GT}$ & $5^{\prime} ; 3^{\prime}$ \\
\hline TC4117 & Elastase 3 precursor & & \\
\hline TC3786 & Elongation factor 1-alpha & & \\
\hline TC3875 & Ependymin & & \\
\hline TC3788 & $\begin{array}{l}\text { Eukaryotic translation initiation factor } \\
3 \text { subunit } 6(48 \mathrm{kD})\end{array}$ & $\mathrm{CA}$ & $3^{\prime}$ \\
\hline TC4023 & Fatty acid-binding protein, liver & & \\
\hline TC4138 & Fatty acyl elongase & $\mathrm{CA}$ & $5^{\prime}$ \\
\hline TC4437 & Fibrinogen alpha chain E variant & & \\
\hline TC3963 & Fibrinogen B-beta subunit precursor & $\mathrm{CA}$ & $3^{\prime}$ \\
\hline
\end{tabular}


Table 3 Continued

\begin{tabular}{|c|c|c|c|}
\hline Contig $^{1}$ & Gene match & Repeat type ${ }^{2}$ & Location $^{3}$ \\
\hline TC3842 & Fibrinogen gamma polypeptide & $\mathrm{CA}$ & $3^{\prime}$ \\
\hline TC4423 & Fish RyR1 isoform & GA, GT & $3^{\prime}$ \\
\hline TC3028 & Fructose-bisphosphate aldolase C & $\mathrm{CA}$ & $3^{\prime}$ \\
\hline TC4040 & GABA-A receptor-associated protein & $\mathrm{CA}, \mathrm{CT}$ & $3^{\prime}$ \\
\hline TC3257 & $\begin{array}{l}\text { GDP-fucose protein O-fucosyltransferase } \\
1 \text { precursor }\end{array}$ & & \\
\hline TC4225 & Gene HMG-T2 protein & GT & $3^{\prime}$ \\
\hline TC4092 & Glutathione S-transferase & $\mathrm{CT}$ & $3^{\prime}$ \\
\hline TC2963 & Glyceraldehyde 3-phosphate dehydrogenase & GT & $3^{\prime}$ \\
\hline TC4457 & G-protein gamma 3 subunit & $\mathrm{CA}$ & $3^{\prime}$ \\
\hline TC4623 & Growth arrest-specific 7-cb protein & & \\
\hline TC3972 & $\begin{array}{l}\text { Growth hormone-releasing hormone/PACAP } \\
\text { precursor }\end{array}$ & $\mathrm{CT} ; \mathrm{CG}$ & $5^{\prime} ; 3^{\prime}$ \\
\hline TC3150 & GTP binding protein Rabla & & \\
\hline TC3302 & GTPase cRhoA & $\mathrm{CA}$ & $3^{\prime}$ \\
\hline TC3033 & Heat shock $90 \mathrm{kD}$ protein 1 alpha & & \\
\hline TC4011 & Helix-loop-helix protein & GT & $3^{\prime}$ \\
\hline TC2966 & Hemoglobin beta chain & & \\
\hline TC4126 & High choriolytic enzyme 1 precursor & & \\
\hline TC4662 & hnRNP-E2 protein & & \\
\hline TC4479 & Homogentisate 12 -dioxygenase & AT & $5^{\prime}$ \\
\hline TC3241 & HT028 & & \\
\hline TC3221 & Hypothetical protein FLJ20625 & AT; GT & $5^{\prime} ; 3^{\prime}$ \\
\hline TC3707 & Hypothetical protein FLJ21963 & $\mathrm{CA}$ & $5^{\prime}$ \\
\hline TC3716 & IP1 & & \\
\hline TC3687 & KIAA0472 protein & $\mathrm{CA}$ & $3^{\prime}$ \\
\hline TC3756 & KIAA0659 protein & $\mathrm{CA}$ & $3^{\prime}$ \\
\hline TC4246 & KIAA1089 protein & & \\
\hline TC3359 & KIAA1208 protein & GT & $3^{\prime}$ \\
\hline TC4417 & KIAA1399 protein & GT; GT & $5^{\prime} ; 3^{\prime}$ \\
\hline TC3605 & KIAA1678 protein & & \\
\hline TC3234 & Lactate dehydrogenase-A & & \\
\hline TC3090 & LanC-like protein 1 & & \\
\hline TC3921 & LD31834p & $\mathrm{CA}, \mathrm{CT}$ & $3^{\prime}$ \\
\hline TC4103 & Legumain precursor & $\mathrm{AGT}, \mathrm{CT}, \mathrm{CA}$ & $3^{\prime}$ \\
\hline TC4129 & LIM/homeobox protein Lhx6.1 & $\mathrm{CT}$ & $3^{\prime}$ \\
\hline TC3513 & Lipoprotein lipase & & \\
\hline TC4236 & $\begin{array}{l}\text { Low molecular weight protein } \\
\text { tyrosine phosphatase isoform A }\end{array}$ & $\mathrm{CA}$ & $3^{\prime}$ \\
\hline TC3149 & MAP kinase kinase 4 & GT & $3^{\prime}$ \\
\hline TC3486 & Metabotropic glutamate receptor 7 precursor & $\mathrm{CA}$ & $3^{\prime}$ \\
\hline TC4141 & Metalloprotease/disintegrin-like protein & $\mathrm{GT}, \mathrm{CT}$ & $3^{\prime}$ \\
\hline TC3837 & MHC class I alpha chain & $\mathrm{CA}$ & $3^{\prime}$ \\
\hline TC3833 & MHC class I alpha chain & GA; CA, TTA & $5^{\prime} ; 3^{\prime}$ \\
\hline TC3011 & MHC class I alpha chain & GA, CA & $3^{\prime}$ \\
\hline TC3857 & MHC class I antigen & GA & $5^{\prime}$ \\
\hline TC4102 & MHC class II antigen & GT & $5^{\prime}$ \\
\hline TC3679 & MHC class II antigen & $\mathrm{CA}$ & $3^{\prime}$ \\
\hline \multirow[t]{2}{*}{ TC3495 } & Mitochondrial import stimulation factor & & \\
\hline & L subunit & CT & $5^{\prime}$ \\
\hline
\end{tabular}


Table 3 Continued

\begin{tabular}{|c|c|c|c|}
\hline Contig $^{1}$ & Gene match & Repeat type ${ }^{2}$ & Location $^{3}$ \\
\hline TC4199 & Myeloid protein-1 & $\mathrm{CA}$ & $3^{\prime}$ \\
\hline TC3062 & NAD kinase & $\mathrm{CA}$ & $5^{\prime}$ \\
\hline TC3048 & $\begin{array}{l}\text { Nascent polypeptide-associated complex, } \\
\text { alpha polypeptide }\end{array}$ & & \\
\hline TC4054 & Neuronal protein NP25 & CA & $5^{\prime}$ \\
\hline TC3050 & Novel protein similar to vertebrate NSF & CA, GT & $3^{\prime}$ \\
\hline TC4237 & Nucleolar RNA helicase II & & \\
\hline TC3199 & Oct2 alpha/beta coding region & ATTC, CA; CT & $3^{\prime}$ \\
\hline TC4123 & P24B PROTEIN PRECURSOR & & \\
\hline TC3101 & Pancreatic carboxypeptidase A1 precursor & & \\
\hline TC4238 & Parvalbumin alpha & $\mathrm{CT}, \mathrm{CA}$ & $5^{\prime}$ \\
\hline TC3170 & Pentraxin & & \\
\hline TC3070 & Phosphofructokinase & GT & $3^{\prime}$ \\
\hline TC3756 & Pleiotrophin 1 & $\mathrm{CA}$ & $3^{\prime}$ \\
\hline TC3239 & $\begin{array}{l}\text { Polyadenylate-binding protein, } \\
\text { testis-enriched isoform }\end{array}$ & & \\
\hline TC3538 & Procarboxypeptidase B & & \\
\hline TC3906 & Protein synthesis factor & CT, GT & $3^{\prime}$ \\
\hline TC3117 & Ran binding protein 1 & GGA; CT & $\mathrm{CDS} ; 3^{\prime}$ \\
\hline TC4234 & Ras-related protein Rab-18 & TTA & $3^{\prime}$ \\
\hline TC4182 & Ras-related protein Rab-2 & $\mathrm{CT}$ & $3^{\prime}$ \\
\hline TC4107 & Ras-related protein Rab-7 & $\mathrm{CA}, \mathrm{CA}$ & $3^{\prime}$ \\
\hline TC3779 & Ribonucleoprotein & & \\
\hline TC4204 & RIKEN cDNA 1110008 F13 gene & $\mathrm{CT}$ & $3^{\prime}$ \\
\hline TC3162 & RNA polymerase $B$ transcription factor 3 & $\mathrm{CA}$ & $5^{\prime}$ \\
\hline TC3481 & rS-Rex-s & $\mathrm{CA}, \mathrm{GT}$ & $3^{\prime}$ \\
\hline TC3343 & Serine protease inhibitor-E2 & & \\
\hline TC3349 & SH3 domain protein $2 \mathrm{~A}$ & CT, GT & $5^{\prime}$ \\
\hline TC3852 & Solute carrier family 25 member 5 protein & GT & $3^{\prime}$ \\
\hline TC3737 & Spermine synthase & GA & $5^{\prime}$ \\
\hline TC3146 & Splicing factor, arginine/serine-rich 1 & $\mathrm{CA}$ & $3^{\prime}$ \\
\hline TC4132 & SPPL2a protein & & \\
\hline TC4350 & Stathmin 3 & & \\
\hline TC3708 & Stress-induced-phosphoprotein 1 & & \\
\hline TC4219 & $\begin{array}{l}\text { Succinate dehydrogenase iron-protein } \\
\text { subunit B }\end{array}$ & & \\
\hline TC3136 & Superior cervical ganglion-10 protein & $\mathrm{CA}$ & $3^{\prime}$ \\
\hline TC4269 & Synaptotagmin I & AAAT & $3^{\prime}$ \\
\hline TC3957 & Synuclein & CT; CA & $5^{\prime} ; 3^{\prime}$ \\
\hline TC4063 & TB2 & & \\
\hline TC3904 & Transcription factor BTF3 & & \\
\hline TC3225 & Transferrin & & \\
\hline TC3846 & Translation elongation factor 2 & & \\
\hline TC3016 & Translation elongation factor EF-1 gamma & & \\
\hline TC3218 & Translation initiation factor $5 \mathrm{~A}$ & GT & $3^{\prime}$ \\
\hline TC3010 & $\begin{array}{l}\text { Translationally-controlled tumor } \\
\text { protein }(\mathrm{TCTP})\end{array}$ & & \\
\hline TC3154 & Transmembrane 4 superfamily member 2 & CTT; CA, TCG & $5^{\prime} ; 3^{\prime}$ \\
\hline TC3405 & Transmembrane protein BRI & & \\
\hline TC4450 & Transposase & $\mathrm{CA}$ & $5^{\prime}$ \\
\hline
\end{tabular}


Table 3 Continued

\begin{tabular}{|c|c|c|c|}
\hline Contig $^{1}$ & Gene match & Repeat type ${ }^{2}$ & Location $^{3}$ \\
\hline TC3307 & $\begin{array}{l}\text { Trifunctional enzyme beta subunit, } \\
\text { mitochondrial precursor }\end{array}$ & $\mathrm{CA}$ & $3^{\prime}$ \\
\hline TC3132 & Triglyceride lipase & $\mathrm{CA}$ & $5^{\prime}$ \\
\hline TC4034 & $\begin{array}{l}\text { Trypsin III cationic precursor } \\
\text { (EC } 3.4 .21 .4 \text { ) }\end{array}$ & & \\
\hline TC3928 & Trypsinogen 1 & & \\
\hline TC3924 & Tubulin alpha-3 chain & & \\
\hline TC4223 & Tubulin beta-1 chain & & \\
\hline TC3206 & Tubulin beta- 4 chain & CA & $5^{\prime}$ \\
\hline TC3226 & Ubiquitin carboxyl-terminal hydrolase & AAAC & $5^{\prime}$ \\
\hline TC4674 & Ubiquitously expressed transcript & & \\
\hline TC4110 & Unnamed protein product gb:BAC04906 & GT, GT & $5^{\prime}$ \\
\hline TC3548 & $\begin{array}{l}\text { Vacuolar ATP synthase } 16 \mathrm{kDa} \\
\text { proteolipid subunit }\end{array}$ & & \\
\hline TC4003 & $\begin{array}{l}\text { Voltage-dependent anion-selective } \\
\text { channel protein } 2\end{array}$ & & \\
\hline TC3187 & $\begin{array}{l}\text { Voltage-gated potassium channel } \\
\text { beta-3 subunit }\end{array}$ & GT & $3^{\prime}$ \\
\hline TC2993 & $\begin{array}{l}\text { Warm-temperature-acclimation-related- } \\
65 \mathrm{kDa} \text {-protein }\end{array}$ & CT & $5^{\prime}$ \\
\hline TC4120 & $\begin{array}{l}\text { Wolf-Hirschhorn syndrome } \\
\text { candidate } 2 \text { protein }\end{array}$ & & \\
\hline TC4038 & WW domain binding protein-2 & GT & $3^{\prime}$ \\
\hline
\end{tabular}

${ }^{1}$ Contigs and individual clone sequences available at http://www.tigr.org/tdb/tgi/cfgi.

${ }^{2}$ Repeats found in $5^{\prime}$ and $3^{\prime}$ end of gene separated by semicolon.

${ }^{3}$ Location with respect to coding region.

(5':BM496042 and $3^{\prime}:$ BM497061) contained a (CA) $)_{17}$ motif, a separate $(C A)_{18}$ motif, and an $(\mathrm{ATTT})_{6}$ motif. Another clone selected with a CA probe (BM495383) contained separate $(\mathrm{CAT})_{8}$ and $(\mathrm{GT})_{12}$ motifs.

Twelve clones that were PCR-positive for CA microsatellites, but contained a repeat too far within the clone to be detected by sequencing with vector primers, were resequenced using $(\mathrm{CA})_{9} \mathrm{CBN}$ and $(\mathrm{GT})_{9} \mathrm{GVN}$ primers. Half of these clones yielded readable sequence from both reactions so genotyping primers could be constructed to amplify across the repeat region. The addition of 5\% DMSO to the sequencing reaction and the use of the dGTP sequencing kit facilitated longer and clearer reads through $\mathrm{GC}$-rich repeat regions.

\section{Repeat Polymorphism}

Sixty-one percent of the initial primer sets tested (13/33) provided amplification products. The success rate increased to $90 \%(53 / 59)$ for subsequent cDNAs when primer design was weighted toward amplification fragments less than $200 \mathrm{bp}$. Sample genotyping revealed $86 \%(20 / 22)$ of these markers were polymorphic in catfish linkage map reference families (Table 4). A minimum number of five repeats was tested and found to be polymorphic. 


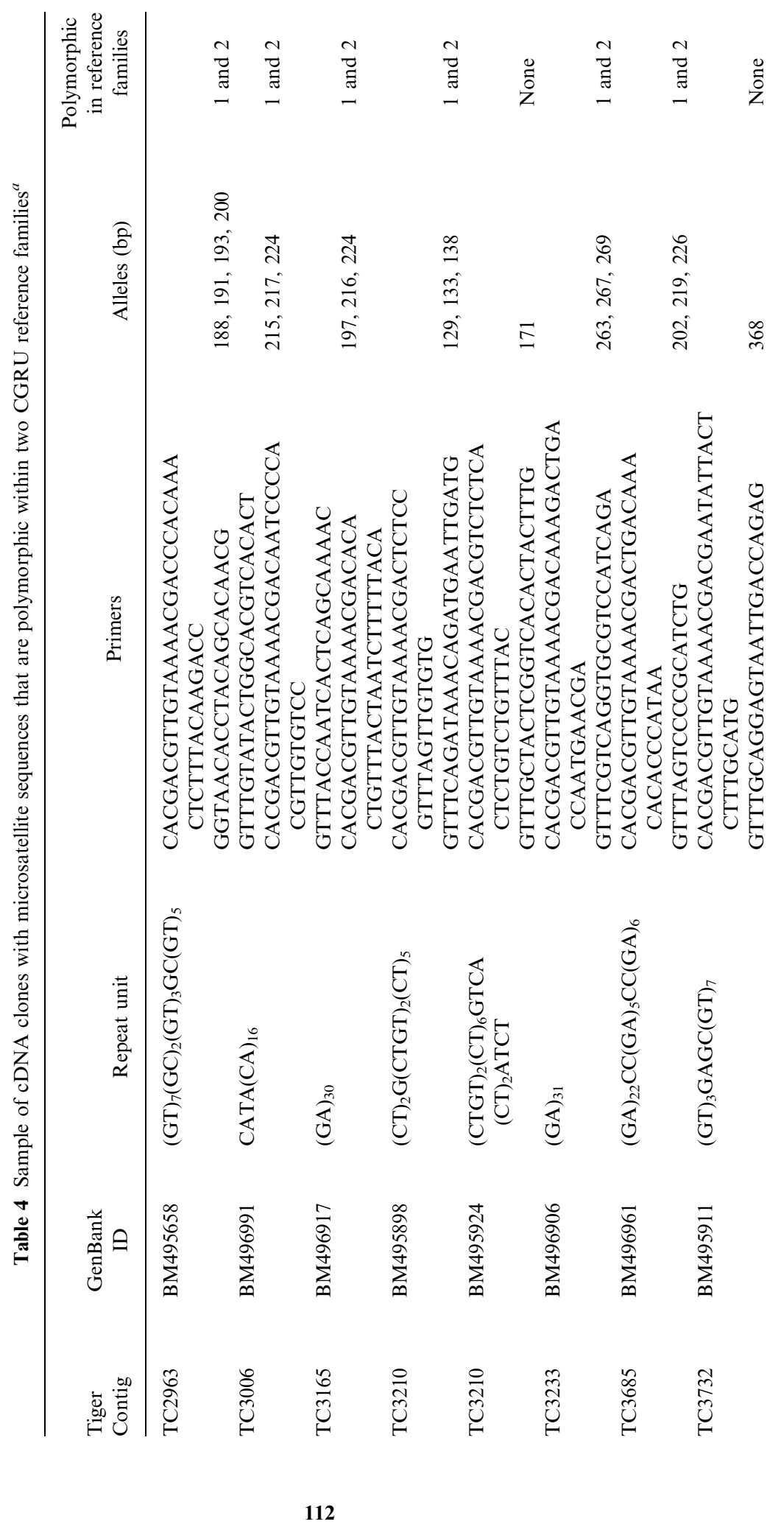




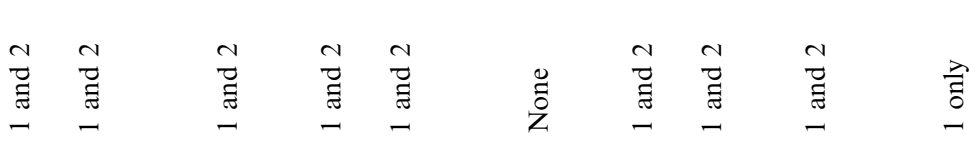

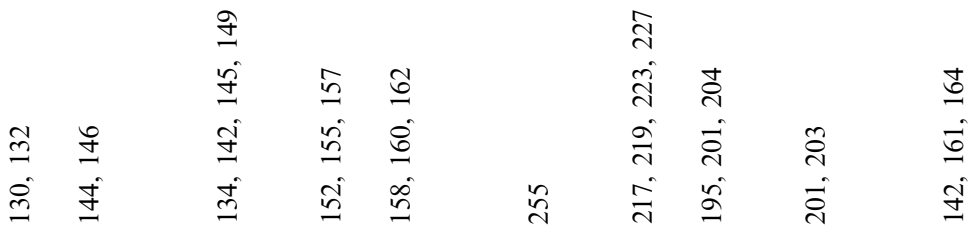

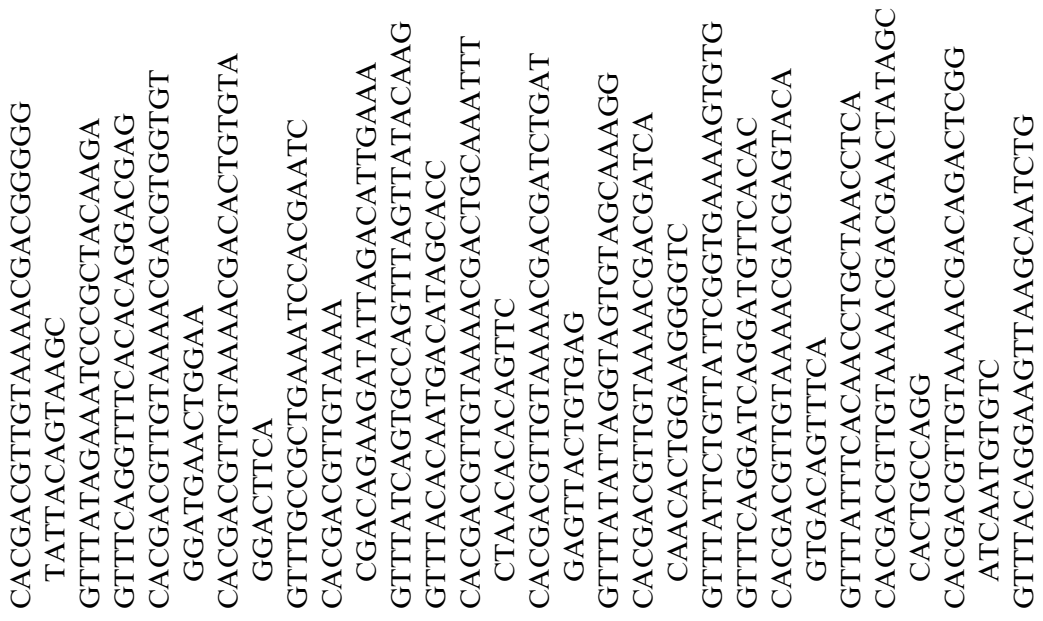

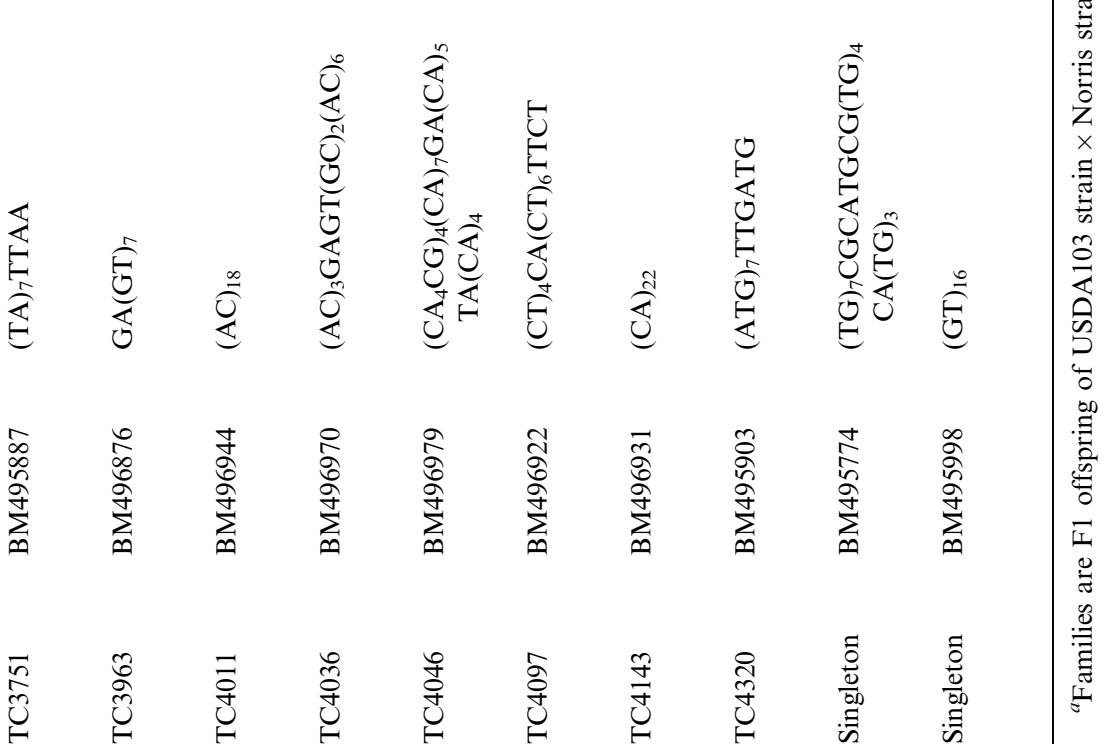




\section{DISCUSSION}

Microsatellite repeats in EST sequences provide a rich source of potentially highly informative markers that can be placed on comparative maps since these sequences can be linked to known loci. The catfish cDNA sequences are unique in that they appear to contain a large proportion of microsatellite-containing EST's compared to other species (21-23). Liu et al. (22) and Karsi et al. (21) reported finding microsatellites in $7 \%$ and $9 \%$ of brain and skin ESTs, respectively. This is much higher than that found in mammalian ESTs and even more than twice the rate in zebrafish ESTs (Table 2). Many of these catfish ESTs contained repeat regions in mammalian orthologues. Since many of the EST sequences of most species are generated primarily from the $5^{\prime}$-end, the catfish orthologues may provide targets for full-length or $3^{\prime}$ sequencing projects to identify microsatellites in other species as well. Sequencing from the $3^{\prime}$ end of the catfish clones was worthwhile in providing additional markers that would not have been discovered using $5^{\prime}$ sequencing alone. Some of the finished sequences did not contain repeats probably because the microsatellites are too deep in the transcript to reach with a single pass, the sequencing failed at repetitive sequences or through very long poly(A) tails, the hybridization probes bound to repeats other than microsatellites, or contamination from abundant clones that were not eliminated from the library. The average insert size of the primary library was $1.8 \mathrm{~kb}$ and would not be covered by $600 \mathrm{bp}$ reads from both ends. The addition of more EST sequencing reads could assemble these reads into complete contigs that contain repeats and provide identification of repeat-containing clones that currently do not have significant Blast hits. As reported for human brain (24) it is possible that cDNA libraries constructed from catfish brain mRNA contain clones corresponding to $5^{\prime}$-truncated transcripts or clones with very long $3^{\prime}$-untranslated regions that would contain microsatellites and not provide a significant Blast hit.

Initial characterization of these clones demonstrated $86 \%$ of the cDNAs could be placed on the catfish genetic linkage map (14). Polymorphism was not entirely dependent on repeat length, since repeats of five dinucleotides were polymorphic while some of six or seven were not. Library enrichment sometimes provided better potential markers because neighboring repeats were longer than the targeted repeat. The presence of introns between the primer locations may have caused some failures for initial PCR testing, but sequencing of longer genomic amplification products, or sequencing of BAC clones, should allow the design of appropriate primers for further marker development. Considering alternative methods for marker development of known loci, this approach is still an efficient means of mapping Type I loci. Genomic sequencing will also allow the discovery of SNPs (25), which can be used to place these genes on the genetic linkage maps and provide specific amplification products for physical mapping. Because these repeats reside in transcribed regions that are primarily single-copy, potential problems of marker development within repetitive sequences in intergenic regions may be reduced.

Many microsatellite sequences began immediately following the $5^{\prime}$ cloning site, which leads to speculation as to whether these microsatellite sequences are associated with transcription start sites or are just a cloning artifact from reverse transcription. Microsatellite polymorphisms in the promoter region of the bovine growth hormone receptor gene and tilapia prolactin gene have been associated with differential 
expression $(26,27)$. However, very little data are available from other species, which contain few microsatellites in expressed cDNAs. Further analysis of these cDNAs could address the potential role of repeat regions in the expression of the protein products of these genes, since repeat sequences in the $5^{\prime}$ UTR can decrease protein expression without affecting levels of mRNA (28).

The enrichment reduced by one-sixth the number of clones required to identify the same number of microsatellite markers in known genes and doubled the number of unique clones with microsatellites. Enrichment did increase the redundancy of the sequenced clones three-fold; however, deeper sequencing of the library would probably produce even more microsatellites in unique clones before redundancy would reduce efficiency. Enrichment increased the probability of finding more markers, and sequencing multiple transcripts from both ends provided more contigs and identified more anchored markers than sequencing the primary library alone. Addition of polymorphic Type I markers to the catfish genetic linkage map will provide comparative map information with other vertebrate species and aid identification of genomic regions controlling economically important production traits.

\section{ACKNOWLEDGMENTS}

The authors gratefully thank the expert assistance of Mary Duke, Debra Harris, Marjorie Jennings, and Mona Kirby; Sherry Kluver for manuscript preparation, and Brent Page, Lanie Bilodeau, and Gary A. Rohrer for critical review of the manuscript. The sequences described here have been submitted to GenBank under the accession numbers BM495166-BM497150.

\section{REFERENCES}

1. Kappes SM. Utilization of gene mapping information in livestock animals. Theriogenol 1999; 51:135-147.

2. Georges M. Towards marker assisted selection in livestock. Reprod Nutri Develop 1999; 39:555-561.

3. Groenen MA, Crooijmans RP, Veenendaal A, Cheng HH, Siwek M, van der Poel JJ. A comprehensive microsatellite linkage map of the chicken genome. Genomics 1998; 49: 265-274.

4. Kappes SM, Keele JW, Stone RT, McGraw RA, Sonstegard TS, Smith TP, LopezCorrales NL, Beattie CW. A second-generation linkage map of the bovine genome. Genome Res 1997; 7:235-249.

5. Rohrer GA, Alexander LJ, Hu Z, Smith TP, Keele JW, Beattie CW. A comprehensive map of the porcine genome. Genome Res 1996; 6:371-391.

6. Ponsuksili S, Wimmers K, Yerle M, Schellander K. Mapping of 93 porcine ESTs preferentially expressed in liver. Mamm Genome 2001; 12:869-872.

7. Rink A, Santschi EM, Eyer KM, Roelofs B, Hess M, Godfrey M, Karajusuf EK, Yerle M, Milan D, Beattie CW. A first-generation EST RH comparative map of the porcine and human genome. Mamm Genome 2002; 13:578-587.

8. Ruyter-Spira CP, de Koning DJ, van der Poel JJ, Crooijmans RP, Dijkhof RJ, Groenen MA. Developing microsatellite markers from cDNA: A tool for adding expressed sequence tags to the genetic linkage map of the chicken. Anim Genet 1998; 29:85-90. 
9. Stone RT, Grosse WM, Casas E, Smith TP, Keele JW, Bennett GL. Use of bovine EST data and human genomic sequences to map 100 gene-specific bovine markers. Mamm Genome 2002; 13:211-215.

10. Moran C. Microsatellite repeats in pig (Sus domestica) and chicken (Gallus domesticus) genomes. J Hered 1993; 84:274-280.

11. Grosse WM, Kappes SM, McGraw RA. Linkage mapping and comparative analysis of bovine expressed sequence tags (ESTs). Anim Genet 2000; 31:171-177.

12. Rohrer GA, Fahrenkrug SC, Nonneman D, Tao N, Warren WC. Mapping microsatellite markers identified in porcine EST sequences. Anim Genet 2002; 33:372-376.

13. Ruyter-Spira CP, Crooijmans RP, Dijkhof RJ, van Oers PA, Strijk JA, van der Poel JJ, Groenen MA. Development and mapping of polymorphic microsatellite markers derived from a chicken brain cDNA library. Anim Genet 1996; 27:229-234.

14. Waldbieser GC, Bosworth BG, Nonneman DJ, Wolters WR. A microsatellite-based genetic linkage map for channel catfish, Ictalurus punctatus. Genetics 2001; 158:727-734.

15. Ewing B, Hillier L, Wendl MC, Green P. Base-calling of automated sequencer traces using phred. I. Accuracy assessment. Genome Res 1998; 8:175-185.

16. Ewing B, Green P. Base-calling of automated sequencer traces using phred. II. Error probabilities. Genome Res 1998; 8:186-194.

17. Chou H-H, Holmes MH. DNA sequence quality trimming and vector removal. Bioinformatics 2001; 17:1093-1104.

18. Altschul SF, Gish W, Miller W, Myers EW, Lipman DJ. Basic local alignment search tool. J Molec Biol 1990; 215:403-410.

19. Rozen S, Skaletsky HJ. Primer3. 1998. Code available at http://www-genome.wi.mit. edu/genome_software/other/primer3.html.

20. Rozen S, Skaletsky HJ. Primer3 on the WWW for general users and for biologist programmers. Methods Molec Biol 2000; 132:365-386.

21. Karsi A, Cao D, Li P, Patterson A, Kocabas A, Feng J, Ju Z, Mickett KD, Liu Z. Transcriptome analysis of channel catfish (Ictalurus punctatus): Initial analysis of gene expression and microsatellite-containing cDNAs in the skin. Gene 2002; 285:157-168.

22. Liu Z, Tan G, Li P, Dunham RA. Transcribed dinucleotide microsatellites and their associated genes from channel catfish Ictalurus punctatus. Biochem Biophys Res Comm 1999; 259:190-194.

23. Liu Z, Li P, Kocabas A, Karsi A, Ju Z. Microsatellite-containing genes from the channel catfish brain: Evidence of trinucleotide repeat expansion in the coding region of nucleotide excision repair gene RAD23B. Biochem Biophys Res Comm 2001; 289:317-324.

24. Seki N, Ohira M, Nagase T, Ishikawa K, Miyajima N, Nakajima D, Nomura N, Ohara O. Characterization of cDNA clones in size-fractionated cDNA libraries from human brain. DNA Res 1997; 4:345-349.

25. Fahrenkrug SC, Freking BA, Smith TP, Rohrer GA, Keele JW. Single nucleotide polymorphism (SNP) discovery in porcine expressed genes. Anim Genet 2002; 33:186-195.

26. Hale CS, Herring WO, Shibuya H, Lucy MC, Lubahn DB, Keisler DH, Johnson GS. Decreased growth in angus steers with a short TG-microsatellite allele in the P1 promoter of the growth hormone receptor gene. J Anim Sci 2000; 78:2099-2104.

27. Streelman JT, Kocher TD. Microsatellite variation associated with prolactin expression and growth of salt-challenged tilapia. Physiol Genom 2002; 9:1-4.

28. Amirhaeri S, Wohlrab F, Wells RD. Differential effects of simple repeating DNA sequences on gene expression from the SV40 early promoter. J Biol Chem 1995; 270:3313-3319. 